\title{
The Current Status of EMDR Therapy Involving the Treatment of Complex Posttraumatic Stress Disorder
}

\author{
Ad de Jongh \\ University of Amsterdam and Vrije Universiteit Amsterdam, Amsterdam, the Netherlands \\ Salford University, Manchester, United Kingdom \\ University of Worcester, Worcester, United Kingdom \\ Queen's University, Belfast, Northern Ireland \\ Iva Bicanic \\ University Medical Center Utrecht, Utrecht, the Netherlands \\ Suzy Matthijssen (1) \\ Altrecht Academic Anxiety Centre, Utrecht, the Netherlands \\ Utrecht University, Utrecht, the Netherlands \\ Benedikt L. Amann \\ Institut de Neuropsiquiatria i Addiccions (INAD), Barcelona, Spain \\ Institut Hospital del Mar d'Investigacions Mèdiques (IMIM), Barcelona, Spain \\ Autonomous University of Barcelona, CIBERSAM, Spain \\ Arne Hofmann \\ EMDR Institut Deutschland, Bergisch Gladbach, Germany \\ Derek Farrell \\ University of Worcester, Worcester, United Kingdom \\ Christopher W. Lee \\ Murdoch University, Perth, Australia \\ University of Western Australia, Perth, Australia \\ Louise Maxfield \\ Ridgetown, Ontario, Canada
}

Complex posttraumatic stress disorder (CPTSD) is a diagnostic entity that will be included in the forthcoming edition of the International Classification of Diseases, 11 th Revision (ICD-11). It denotes a severe form of PTSD, comprising not only the symptom clusters of PTSD (Diagnostic and Statistical Manual of Mental Disorders, Fourth Edition [DSM-IV-TR]), but also clusters reflecting difficulties in regulating emotions, disturbances in relational capacities, and adversely affected belief systems about oneself, others, or the world. Evidence is mounting suggesting that first-line trauma-focused treatments, including eye movement desensitization and reprocessing (EMDR) therapy, are effective not only for the treatment of PTSD, but also for the treatment of patients with a history of early childhood interpersonal trauma who are suffering from symptoms characteristic of CPTSD. However, controversy exists as to when EMDR therapy should be offered to people with CPTSD. This article reviews the evidence in support of EMDR therapy as a first-line treatment for CPTSD and addresses the fact that there appears to be little empirical evidence supporting the view that there should be a stabilization phase prior to trauma processing in working with CPTSD. 
Keywords: complex posttraumatic stress disorder (CPTSD); eye movement desensitization and reprocessing (EMDR) therapy; stabilization; three-phase model; interpersonal childhood trauma

A ccording to international guidelines in the field of psychotraumatology, posttraumatic stress disorder (PTSD) should be treated with empirically supported therapies; for example, traumafocused cognitive behavioral therapy or eye movement desensitization and reprocessing (EMDR) therapy (e.g., Department of Veterans Affairs and the Department of Defense, 2017; International Society of Traumatic Stress Studies, 2018a; World Health Organization, 2013). Trauma-focused cognitive behavioral therapy includes exposure (imaginal or prolonged) and cognitive approaches. Although there is consensus about the application of these therapies for patients with PTSD, there is less agreement in the field about its (timing of the) use in individuals suffering from the consequences of early traumatization (Cloitre et al., 2011, 2012), in particular those suffering from "Complex PTSD," or CPTSD. This term is widely used for a specific variant of PTSD involving a broad diversity of symptoms that is purported to characterize the impact of repeated and prolonged interpersonal traumatization in early childhood (Cloitre et al., 2012; Van der Kolk, Roth, Pelcovitz, Sunday, \& Spinazzola, 2005). The purpose of this article is to provide a brief narrative overview of the current knowledge about CPTSD and the evidence base regarding the treatment of this condition using EMDR therapy.

\section{Complex Trauma or CPTSD?}

There is some confusion among clinicians about the terminology of complex trauma and CPTSD. What is meant by the term "complex"? Is it referring to the type of traumatic event, the consequences of the trauma, the need for a multiphased treatment, or the difficulties involving the processing of the traumatic memories? The term "complex trauma" refers to experienced traumatic events that are interpersonal, long-term, repeated, and severe (American Psychiatric Association, 2013, p. 276), while the term CPTSD refers to a symptom-focused, diagnostic entity. Although it was decided not to include CPTSD in the latest version of the Diagnostic and Statistical Manual of Mental Disorders, Fifth Edition (DSM-5; APA, 2013; Resick et al., 2012), CPTSD will be included in the forthcoming classification system of the World Health Organization (International Classification of
Diseased 11th Revision, ICD-11; 2018a). In the criteria used by International Classification of Diseases, 11 th Revision (ICD-11), CPTSD is diagnosed when the core criteria of PTSD (DSM-IV-TR, i.e., re-experiences, avoidance behavior, and hyperarousal) are met and additional symptoms of emotion regulation problems, disturbance of relational skills, and negative core beliefs are present (Cloitre et al., 2013, Maercker et al., 2013). A key point to note is that individuals with CPTSD by definition need to meet the diagnostic criteria of PTSD. Therefore, if the traumatic memories that fuel the core PTSD symptoms are successfully processed, then the person no longer fulfils the diagnostic criteria of CPTSD. Given it is a new classification, a clinical interview to formally establish a CPTSD diagnosis has become available only in the last few years (Hyland et al., 2017).

There is a widespread misunderstanding that people who have experienced complex trauma would be likely to meet the diagnostic criteria of PTSD or CPTSD (see Chen et al., 2010; Ter Heide, Mooren, \& Kleber, 2016). However, other psychiatric disorders, including a lifetime diagnosis of anxiety disorders, depression, eating disorders, sleep disorders, and selfdestructive behavior such as attempted suicide seem to be more likely to develop as a result of sexual abuse than PTSD per se (Chen et al., 2010). One of the few studies that examined the relationship between childhood sexual abuse and CPTSD found that only a quarter of the abused women met the classification criteria of CPTSD (McLean, Toner, Jackson, Desrocher, \& Stuckless, 2006).

\section{The Role of Stabilization in the Treatment of CPTSD}

In 2012, the International Society of Traumatic Stress Studies (ISTSS) published their first guidelines regarding the treatment of CPTSD. The guideline committee recommended that the treatment of people with early childhood interpersonal trauma and symptoms of CPTSD should be phased, according to the so-called three-phase model (Cloitre et al., 2012). This treatment approach started with a stabilization phase, followed by the second treatment phase, which entailed the processing of the traumatic memories using an evidence-based (trauma-focused) therapy. The third and final treatment phase focused on "reintegration," 
(re)participation in life in general and the resumption of daily activities (Cloitre et al., 2012).

The goals of the stabilization phase are generally to enhance safety, establish a therapeutic relationship, and teach stress management and affect regulation skills, by offering psychoeducation about the consequences of the traumatic events and by applying cognitive restructuring of beliefs involving selfblame (Cloitre, Koenen, Cohen, \& Han, 2002). The idea of starting with a stabilization phase in patients with CPTSD prior to the confrontation with their traumatic memories can be traced back to the assumption that people who have experienced interpersonal traumatization in their youth, and consequently experience symptoms of CPTSD, lack the psychological stability to be confronted with their traumatic memories and to bear the emotional arousal that is related to this (e.g., Janet \& Psychological Healing, 1919). Accordingly, it is a widely held belief by clinicians that premature confrontation with emotionally charged memories, at a time when the patient does not (yet) possess the skills to cope with it, could lead to negative effects such as aggravation of symptoms, psychological disruption, and suicidality (Cloitre et al., 2011). Following this line of reasoning, in order not to directly overwhelm patients with the traumatic images, cognitions, and emotions, patients in a stabilization phase first acquire skills in the field of self-control and affect regulation (Dorrepaal, Thomaes, \& Draijer, 2006; Zlotnick et al., 1997).

Besides the fact that implementing a stabilization phase in the treatment of CPTSD may have positive effects on patients' motivation for the trauma-related part of the treatment (Cloitre et al., 2011; Kezelman $\&$ Stavropoulos, 2012), there is also evidence suggesting that it is effective in reducing PTSD symptoms (e.g., Cloitre et al., 2010; Eichfeld et al., 2018). However, until now studies that examined the efficacy of stabilization in individuals with CPTSD failed to provide evidence that a phase-based approach is necessary for positive treatment outcomes, or that individuals would profit significantly more from traumafocused treatments when preceded by a stabilization phase (De Jongh et al., 2016). Therefore, it has been recommended that trauma-focused therapies should routinely be offered to adults with CPTSD, consistent with current general PTSD treatment guidelines, and without a preceding stabilization phase (De Jongh et al., 2016). Accordingly, in their recently released version of the treatment guidelines for CPTSD, the ISTSS guideline committee proposed a "personalized medicine" approach in which the focus is put on "the identification of symptoms that are clinically significant (e.g., are severe or associate with functional impairment) to a particular patient and tailoring interventions or series of interventions to address these problems" (ISTSS, 2018b, p. 3). Although the guideline committee recognizes that for patients with CPTSD, interventions aimed at stability and symptom management may be needed, they state that treatment should primarily be focused on facilitating the processing of the childhood memories.

\section{Effectiveness of Trauma-Focused Treatments for Individuals With Symptoms of CPTSD}

At this time, no studies have been published that used a valid measure for assessing patients with the newly developed CPTSD diagnosis. However, a few years ago a broad group of international trauma experts carried out an extensive review of the available literature on the effects of treatments for patients suffering from symptoms that are characteristic of CPTSD, including emotion regulation problems, disturbances in relationships, and the presence of a negative self-concept (De Jongh et al., 2016). They could not find any support for the notion that adults with symptoms characteristic of CPTSD would not respond to traumafocused interventions, nor that these interventions should be preceded by a stabilization phase. These findings were supported by the results of a recent systematic review and meta-analysis of 51 randomized controlled trials of psychological interventions for PTSD with individuals reporting clinically significant levels of one or more CPTSD symptom clusters (Karatzias et al., 2019). The results showed that applying trauma-focused treatments is effective in treating symptoms of CPTSD. While the authors of this metaanalysis found few studies that reported on the effects on affect dysregulation, they found moderate-large to large positive effects on negative self-concept, and moderate to moderate-large positive effects on disturbed relationships.

Effectiveness of EMDR Therapy for Individuals With PTSD Symptoms From Childhood Trauma

Given that the majority of patients with symptoms of CPTSD report a history of interpersonal trauma in childhood, it is relevant to examine whether individuals who have been exposed to early childhood trauma and abuse would be more refractory in the sense that memories of those events are more difficult to recall 
and more difficult to process. To investigate whether treatment of the negative effects of childhood trauma would be disproportionately problematic, Ehring, Morina, Wicherts, Freitag, and Emmelkamp (2014) used a meta-analytic app roach and found that this was not the case. It also provided evidence that PTSD in these patients can be treated effectively according to the general treatment guidelines for PTSD, using trauma-focused therapies. As to EMDR therapy this meta-analysis included three randomized controlled trials that evaluated the effects of EMDR for participants with PTSD symptoms as a consequence of childhood trauma (Edmond \& Rubin, 2004; Scheck, Schaeffer, \& Gillette, 1998; Van der Kolk et al., 2007). The mean effect size of EMDR therapy in these studies was moderate to high not only for PTSD symptom severity, but also for symptoms of depression, anxiety, and dissociation. Ehring's et al. (2014) meta-analysis showed that EMDR therapy in adult survivors of childhood abuse is efficacious. It is however important to note that Van der Kolk et al. (2007), who compared the efficacy of EMDR with fluoxetine and a pill placebo condition, reported that PTSD symptom reduction was significantly less in EMDR participants with childonset trauma compared to those with adult-onset trauma; that is, $33.3 \%$ versus $75.0 \%$ achieved asymptomatic end-state functioning at 6-month follow-up. Unfortunately, it is unclear how many memories of individuals' index traumas were treated. Therefore, it cannot be ruled out that those with child-onset trauma had a higher level of trauma exposure and, consequently, a higher number of memories that needed to be reprocessed compared to their adult-onset counterparts. Hence, particularly for the patients with child-onset memories, a course of six trauma-focused sessions may have been insufficient to process all memories that drove the PTSD symptoms.

More recently, a study into the efficacy of traumafocused treatment of PTSD among patients with schizophrenia or psychosis, found EMDR therapy to be effective (Van den Berg et al., 2015). A history of sexual abuse was reported by $67 \%$ of the sample, and $36.4 \%$ of those treated with EMDR identified multiple incidents of childhood sexual abuse before the age of 12 years. Following the eight treatment sessions, $60 \%$ of the participants in the EMDR condition achieved loss of PTSD diagnosis. These effects remained stable at 12-month follow-up (Van den Berg et al., 2018).

Similar positive results were reported in two EMDR randomized controlled trials with children who had been sexually abused (Jaberghaderi, Greenwald, Rubin, Zand, \& Dolatabadi, 2004) and children with PTSD who had been abused and/or were living in "socially exposed" homes (Ahmad, Larsson, \& Sundelin-Wahlsten, 2007). These EMDR therapies did not include a stabilization phase and the primary focus of treatment was trauma processing.

\section{Safety of Trauma-Focused Treatments for Individuals With Symptoms of CPTSD}

Besides support for the effectiveness of traumafocused treatments, there are no indications that these interventions would have unacceptable risks for patients undergoing them (De Jongh et al., 2016). This notion is perhaps most strongly supported by the results of the study that investigated the safety and effectiveness of EMDR therapy in individuals with a psychotic disorder and comorbid PTSD (Van den Berg et al., 2015). In this study no stabilization phase was offered prior to the trauma treatment, and also the safe-place exercise-assumed to help stabilize patients-was removed from the standard EMDR protocol. Deterioration did occur in some individuals; however, this was four times more likely in the treatment as usual group (four patients) rather than in the EMDR therapy condition group (one patient).

Hence, disruption or permanent worsening of symptoms is not a phenomenon that occurs frequently during trauma-focused treatment in patients with complex trauma presentations, even if a stabilization phase is lacking (Van den Berg et al., 2016; Van Minnen, Harned, Zoellner, \& Mills, 2012). This seems to hold even more true for intensive treatment of this target group. Some recent studies investigated the treatment effects of a treatment format of 8 days combining EMDR ( 8 sessions of 90 minutes) and imaginal exposure with in vivo exposure (8 sessions of 90 minutes), physical activity, and psychoeducation (Bongaerts, Van Minnen, \& De Jongh, 2017; Van Woudenberg et al., 2018; Wagenmans, Van Minnen, Sleijpen, \& De Jongh, 2018; Zoet et al., 2018). The results of these studies indicate that of the patients who suffered from CPTSD with a broad variety of trauma histories and multiple comorbidities who completed this treatment format, more than $80 \%$ showed a clinically meaningful response, while more than half of them lost their PTSD diagnosis following these 8 days of treatment (Van Woudenberg et al., 2018). The finding that only $3.8 \%$ of the patients showed a clinically meaningful increase in PTSD symptoms suggests that an intensive treatment is an effective and safe treatment alternative for patients with symptoms of CPTSD yielding relative high effect sizes and remarkably favorable retention rates of approximately 97\% (Van Woudenberg et al., 2018). 


\section{Clinical Implications}

The research findings outlined in this article suggest that working immediately and directly on traumatic memories can be very helpful for patients with CPTSD and that-fully in line with EMDR's AIP model-resolving symptoms of PTSD may reduce patients' interpersonal challenges, affect dysregulation, and negative self-concept. Nevertheless, from a clinical point of view, there are a number of challenges when treating patients with CPTSD in comparison with those suffering from PTSD. Many fear confronting their memories and emotions. They may be reluctant to focus on their past, and experience impulses to flee, avoid, or distract from their trauma history. Some may shut down or dissociate which limits access to the trauma memories during the sessions. Furthermore, cognitive distortions, for instance, about responsibility or emotions such as survivor guilt, may impede desensitization to the trauma memory. However, instead of addressing these issues with stabilization strategies, they can be directly targeted. For example, EMDR therapy can target the fear of decompensation due to facing a trauma memory or provide cognitive interweaves to influence patients' blocking beliefs and dysfunctional thinking such as self-blame and lack of self-compassion.

There are other CPTSD factors to take into consideration, such as "self-concept" and "relational factors." Effective therapy works best when adjusted to the patient's needs rather than making the patient fit a particular therapy paradigm. As a consequence this may require trauma stabilization and resource installation. In EMDR therapy, trauma processing requires the patient to be connected with the trauma memory-if the patient is not connected, then other clinical interventions would be required, including psychoeducation and resourcing. Moreover, in the case of ongoing treatment it remains important that in between sessions the needs of CPTSD patients are being well monitored, and that, especially in an outpatient setting, supportive interventions should be offered if necessary.

Two important limitations need to be mentioned. One needs to take into account that data on the effective treatment of CPTSD are still scarce, that clinical settings differ, and that social and cultural circumstances may influence the effectiveness of interventions. Furthermore, in cannot be ruled out that patients with CPTSD who participate in traumafocused research represent a subsample of this clinical population who are more prepared to engage in trauma processing than other CPTSD patients in general. Such patients may never volunteer for such research or decline to participate when it is offered.

\section{Conclusion}

Evidence is mounting that trauma-focused treatments, including EMDR therapy, are effective in people with (symptoms of) CPTSD. Further, the efficacy of these treatments is minimally affected by whether the trauma was an early childhood interpersonal trauma, or if the person suffers from severe dissociative or other characteristic symptoms of CPTSD (Ehring et al., 2014; De Jongh et al., 2016; Van Minnen et al., 2016, Van Woudenberg et al., 2018; Wagenmans et al., 2018, Zoet et al., 2018).

Clearly, future research on the treatment of CPTSD is desperately needed, in particular studies that include individuals who have a long history of psychiatric (inpatient) treatments. Fortunately, well-designed studies directly comparing EMDR therapy or other trauma-focused treatments, with and without a preceding stabilization phase in patients with CPTSD with long-lasting histories of interpersonal trauma, are currently under way (e.g., Van Vliet, Huntjens, Van Dijk, \& De Jongh, 2018), and their results will become available in the coming years. These research findings might provide the long-awaited and crucial answer to the question whether there are certain patient groups who are likely to benefit more from stability management and resource development than from standard trauma-focused EMDR therapy.

\section{References}

Ahmad, A., Larsson, B., \& Sundelin-Wahlsten, V. (2007). EMDR treatment for children with PTSD: Results of a randomized controlled trial. Nordic Journal of Psychiatry, 61(5), 349-354. doi:10. 1080/ 08039480701643464

American Psychiatric Association. (2013). Diagnostic and statistical manual of mental disorders (5th ed.). Arlington, VA: American Psychiatric Publishing.

Bongaerts, H., Van Minnen, A., \& De Jongh, A. (2017). Intensive EMDR to treat PTSD patients with severe comorbidity: A case series. Journal of EMDR Practice and Research, 11, 84-95. doi:10.1891/1933-3196.11.2.84

Chen, L. P., Murad, M. H., Paras, M. L., Colbenson, K. M., Sattler, A. L., Goranson, E. N., . . Zirakzadeh, A. (2010). Sexual abuse and lifetime diagnosis of psychiatric disorders: Systematic review and meta-analysis. Mayo Clinic Proceedings, 85(7), 618-629. doi:10.4065/mcp.2009.0583

Cloitre, M., Courtois, C. A., Charuvastra, A., Carapezza, R., Stolbach, B. C., \& Green, B. L. (2011). Treatment 
of complex PTSD: Results of the ISTSS expert clinician survey on best practices. Journal of Traumatic Stress, 24, 615-627.

Cloitre, M., Courtois, C. A., Ford, J. D., Green, B. L., Alexander, P., \& Briere, J. (2012). The ISTSS expert consensus treatment guidelines for complex PTSD in adults. Retrieved from http://www.istss.org/AM/ Template. cfm?Section=ISTSS_Complex_PTSD Treatment_Guidelines \& Template $=/ \mathrm{CM} /$ Content Display.cfm\&ContentID $=5185$

Cloitre, M., Garvert, D. W., Brewin, C. R., Bryant, R. A., \& Maercker, A. (2013). Evidence for proposed ICD11 PTSD and complex PTSD: A latent profile analysis. European Journal of Psychotraumatology, 4, 74-78. doi:10.3402/ ejpt.v4i0.20706

Cloitre, M., Koenen, K. C., Cohen, L. R., \& Han, H. (2002). Skills training in affective and interpersonal regulation followed by exposure: A phase-based treatment for PTSD related to childhood abuse. Journal of Consulting and Clinical Psychology, 70, 1067-1074. doi:10.1037/0022006X.70.5.1067

Cloitre, M., Stovall-McClough, K. C., Nooner, K., Zorbas, P., Cherry, S., \& Jackson, C. L. (2010). Treatment for PTSD related to childhood abuse: A randomized controlled trial. American Journal of Psychiatry, 167, 915-924. doi:10.1176/appi.ajp.2010.09081247.

De Jongh, A., Resick, P. A., Zoellner, L. A., Van Minnen, A., Lee, C. W., Monson, C. M., .. . Bicanic, I. A. E. (2016). A critical analysis of the current treatment guidelines for complex PTSD in adults. Depression and Anxiety, 33, 59-369. doi:10.1002/ da.22469.

Department of Veterans Affairs and the Department of Defense. (2017). A/DoD clinical practice guideline for the management of posttraumatic stress disorder and acute stress disorder. Retrieved from https:// www.healthquality.va.gov/guidelines/ $\mathrm{MH} / \mathrm{ptsd} / \mathrm{VAD}$ oDPTSDCPGFinal012418.pdf

Dorrepaal, E., Thomaes, K., \& Draijer, P. J. (2006). Stabilisatiecursus als antwoord op complexe post traumatische stress stoornis [Stabilization course as an answer to complex posttraumatic stress disorder]. Tijdschrift voor Psychiatrie, 48, 217-222.

Edmond, T. E., \& Rubin, A. (2004). Assessing the long-term effects of EMDR: Results from an 18-month follow-up study with adult female survivors of CSA. Journal of Child Sexual Abuse, 13, 69-86. doi:10.1300/J070v13n01_04

Ehring, T., Morina, W. R., Wicherts, N., Freitag, J. M., \& Emmelkamp, P. M. G. (2014). Meta-analysis of psychological treatments for posttraumatic stress disorder in adult survivors of childhood abuse. Clinical Psychology Review, 34, 645-657. doi:10.1016/j.cpr.2014.10.004

Eichfeld, C., Farrell, D., Mattheß, M., Bumke, P., Sodemann, U., Ean, N., . . . Mattheß, H. (2018). Trauma stabilisation as a sole treatment intervention for post-traumatic stress disorder in Southeast Asia. Psychiatric quarterly, 90(1), 63-88. doi:10.1007/s11126-018-9598-Z
Hyland, P., Shevlin, M., Brewin, C. R., Cloitre, M., Downes, A. J., Jumbe, S., . . Roberts, N. P. (2017). Validation of post-traumatic stress disorder (PTSD) and complex PTSD using the International Trauma Questionnaire. Acta Psychiatrica Scandinavia, 136, 313-322. doi:10. 1111 / acps. 12771

ISTSS Guidelines Committee. (2018a). Guidelines position paper on complex PTSD in adults. Oakbrook Terrace, IL: Author. Retrieved from http://www. istss.org/getattachment/Treating-Trauma/New-ISTSSPrevention-and-Treatment-Guidelines/ISTSS_CPTSDPosition-Paper-(Adults)_FNL.pdf.aspx

ISTSS Guidelines Committee. (2018b). Posttraumatic stress disorder prevention and treatment guidelines methodology and recommendations. Oakbrook Terrace, IL: Author. Retrieved from http: / / www.istss.org/treating-trauma/ new-istss-prevention-and-treatment-guidelines.aspx

Jaberghaderi, N., Greenwald, R., Rubin, A., Zand, S. O., \& Dolatabadi, S. (2004). A comparison of CBT and EMDR for sexually abused Iranian girls. Clinical Psychology and Psychotherapy, 11(5), 358-368. doi:10.1002/ cpp. 395

Janet, P., \& Psychological Healing. (1919). Original publication: Les medications psychologiques (Vol. 1, 2). New York, NY; Paris: Macmillan; Felix Alcan.

Karatzias, T., Murphy, P., Cloitre, M., Bisson, J., Roberts, N., Shevlin, M., . . . Hutton, P. (2019). Psychological interventions for ICD-11 complex PTSD symptoms: Systematic review and meta-analysis. Psychological Medicine, 1-15. doi:10.1017/S0033291719000436

Kezelman, C., \& Stavropoulos, P. (2012). Practice guidelines for treatment of complex trauma and trauma informed care and service delivery. Adults surviving child abuse, Kirribilli. Retrieved from https: / www.recoveryonpurpose.com/upload/ASCA_ Practice\%20Guidelines\%20for\%20the\%20Treatment\% 20of\%20Complex\%20Trauma.pdf

Maercker, A., Brewin, C. R., Bryant, R. A., Cloitre, M., Reed, G. M., \& Van Ommeren, M. (2013). Proposals for mental disorders specifically associated with stress in the International Classification of Diseases-11. The Lancet, 381, 1683-1685. doi:10.1016/S0140-6736(12)62191-6

McLean, L. M., Toner, B., Jackson, J., Desrocher, M., \& Stuckless, N. (2006). The relationship between childhood sexual abuse, complex post-traumatic stress disorder and alexithymia in two outpatient samples: Examination of women treated in community and institutional clinics. Journal of Child Sexual Abuse, 15(3), 1-17. doi:10.1300/J070v15n03_01

Resick, P. A., Bovin, M. J., Calloway, A. L., Dick, A., King, M. W., Mitchell, K. S., . . . Wolf, E. J. (2012). A critical evaluation of the complex PTSD literature: Implications for DSM-5. Journal of Traumatic Stress, 25, 241-251. doi: $10.1002 /$ jts.21699

Scheck, M. M., Schaeffer, J. A., \& Gillette, C. (1998). Brief psychological intervention with traumatized young women: The efficacy of eye movement desensitization 
and reprocessing. Journal of Traumatic Stress, 11, 25-44. doi:10.1023/ A:1024400931106

Ter Heide, F. J. J., Mooren, T. M., \& Kleber, R. J. (2016). Complex PTSD and phased treatment in refugees: A debate piece. European Journal of Psychotraumatology, 7, 28687. doi:10.3402/ ejpt.v7.28687

Van den Berg, D. P. G., De Bont, P. A. J. M., Van de Vleugel, B. M., De Roos, C., De Jongh, A., Van Minnen, A., \& Van der Gaag, M. (2015). Prolonged exposure versus eye movement desensitization and reprocessing versus waiting list for posttraumatic stress disorder in patients with a psychotic disorder: A randomized clinical trial. JAMA Psychiatry, 72, 259-267. doi:10.1001/jamapsychiatry.2014.2637

Van den Berg, D. P., De Bont, P. A., Van der Vleugel, B. M., De Roos, C., De Jongh, A., Van Minnen, A., \& Van der Gaag, M. (2016). Trauma-focused treatment in PTSD patients with psychosis: Symptom exacerbation, adverse events, and revictimization. Schizophrenia Bulletin 42(3), 693-702. doi:10.1093/schbul/sbv172

Van den Berg, D. P. G., Bont, P. A. J. M., Van de Vleugel, B. M., De Roos, C., De Jongh, A., Van Minnen, A., \& Van der Gaag, M. (2018). Long-term outcomes of trauma-focused treatment in psychosis. British Journal of Psychiatry, 212, 180-182. doi:10.1192/bjp.2017.30

Van der Kolk, B. A., Roth, S., Pelcovitz, D., Sunday, S., \& Spinazzola, J. (2005). Disorders of extreme stress: The empirical foundation of a complex adaptation to trauma. Journal of Traumatic Stress, 18, 389-399. doi:10.1002/jts.20047

Van der Kolk, B. A., Spinazzola, J., Blaustein, M. E., Hopper, J. W., Hopper, E. K., \& Simpson, W. B. (2007). A randomized clinical trial of eye movement desensitization and reprocessing (EMDR), fluoxetine, and pill placebo in the treatment of posttraumatic stress disorder: Treatment effects and long-term maintenance. Journal of Clinical Psychiatry, 68, 37-46. doi:10.4088/jcp.v68n0105

Van Minnen, A., Harned, M. S., Zoellner, L., \& Mills, K. (2012). Examining potential contraindications for prolonged exposure therapy for PTSD. European Journal of Psychotraumatology, 3, 1-14. doi:10.3402/ ejpt.v3i0.18805

Van Minnen, A., Van der Vleugel, B. M., Van den Berg, D. P. G., De Bont, P. A. J. M., De Roos, C., Van der Gaag, M., . . . De Jongh, A. (2016). Effectiveness of traumafocused treatment for patients with psychosis with and without the dissociative subtype of post-traumatic stress disorder. British Journal of Psychiatry, 209, 347-348. doi:10.1192/bjp.bp.116.185579

Van Vliet, N.I., Huntjens, R.J.C., Van Dijk, M.K., De Jongh, A. (2018). Phase-based treatment versus immediate trauma-focused treatment in patients with childhood trauma-related posttraumatic stress disorder: Study protocol for a randomized controlled trial. Trials, 19, 138. https: / / doi.org/10.1186/s13063-018-2508-8

Van Woudenberg, C., Voorendonk, E. M., Bongaerts, H., Zoet, H. A., Verhagen, M., Van Minnen, A., . . . De Jongh, A. (2018). The effectiveness of an intensive treatment programme combining prolonged exposure and EMDR for severe posttraumatic stress disorder . European Journal of Psychotraumatology, 9(1). https: / / doi.org/10.1080/20008198.2018.1487225

Wagenmans, A., Van Minnen, A., Sleijpen, M., \& De Jongh, A. (2018). The impact of childhood sexual abuse on the outcome of intensive trauma-focused treatment for PTSD. European Journal of Psychotraumatology, 9(1), 1430962. doi:10.1080/20008198.2018.1430962

World Health Organization. (2013). Guidelines for the management of conditions that are specifically related to stress. Geneva: Author.

Zlotnick, C., Shea, M. T., Rosen, K., Simpson, E., Mulrenin, K., Begin, A., \& Pearlstein, T. (1997). An affectmanagement group for women with posttraumatic stress disorder and histories of childhood sexual abuse. Journal of Traumatic Stress, 10, 425-436.

Zoet, H. A., Wagenmans, A., Van Minnen, A., Van Sleijpen, M., \& De Jongh, A. (2018). Presence of the dissociative subtype of PTSD does not moderate the outcome of intensive trauma-focused treatment for PTSD. European Journal of Psychotraumatology, 9(1). doi:10.1080/20008198.2018.1468707

Disclosure. A. d. J. receives income from published books on EMDR therapy and for the training of postdoctoral professionals in this method. B.L.A presents in national and international EMDR conferences and is head of The European EMDR Research Committee. A.H. receives income from book publications on EMDR and training licensed professionals in this method. C.L. receives fees for providing training in trauma therapies at workshops and conferences. L.M. receives income from a published book on EMDR therapy and is the editor-in-chief of this journal. The other authors have no relevant financial interest or affiliations with any commercial interests related to the subjects discussed within this article.

Correspondence regarding this article should be directed to Ad de Jongh, Academic Centre for Dentistry Amsterdam (ACTA) Gustav Mahlerlaan 3004, 1081 LA Amsterdam, the Netherlands. E-mail: a.de.jongh@acta.nl 